\title{
Experimental investigation of global structures in an incompressible cavity flow using time-resolved PIV
}

\author{
J. Basley $\cdot$ L. R. Pastur • F. Lusseyran • \\ T. M. Faure $\cdot$ N. Delprat
}

Received: 15 January 2010/Revised: 7 July 2010/Accepted: 15 July 2010/Published online: 4 August 2010

(C) The Author(s) 2010. This article is published with open access at Springerlink.com

\begin{abstract}
Open-cavity flows are known to exhibit a few well-defined peaks in the power spectral distribution of velocity or pressure signals recorded close to the impinging corner. The measured frequencies are in fact common to the entire flow, indicating some global organisation of the flow. The modal structures, i.e. the spatial distribution of the most characteristic frequencies in the flow, are experimentally investigated using time-resolved particle image velocimetry. Each spatial point, of the resulting twodimension-two-component (2D-2C) velocity fields, provides time-resolved series of the velocity components $V_{x}$ and $V_{y}$, in a $(x, y)$ streamwise plane orthogonal to cavity bottom. Each local time-series is Fourier-transformed, such as to provide the spectral distribution at any point of the PIV-plane. One finally obtains the spatial structure associated with any frequency of the Fourier spectrum. Some of the modal spatial structures are expected to represent the nonlinear saturation of the global modes, against which the stationary solution of the Navier-Stokes equations may have become linearly unstable. Following Rowley et al.
\end{abstract}

Electronic supplementary material The online version of this article (doi:10.1007/s00348-010-0942-9) contains supplementary material, which is available to authorized users.

J. Basley $(\bowtie) \cdot$ L. R. Pastur · F. Lusseyran ·

T. M. Faure · N. Delprat

LIMSI-CNRS, BP 133, F-91403 Orsay Cedex, France

e-mail: jeremy.basley@limsi.fr

J. Basley · L. R. Pastur

Université Paris, Sud 11, F-91405 Orsay Cedex, France

T. M. Faure $\cdot$ N. Delprat

Université Pierre et Marie Curie, 4 place Jussieu,

F-75005 Paris, France
(J Fluid Mech 641:115-127, 2009), our experimental modal structures may even correspond to the Koopman modes of this incompressible cavity flow.

\section{Introduction}

Since the pioneering work of Zebib (1987) and Jackson (1987), and developments by Mamum and Tuckerman (2009) and Gadoin et al. (2001), global-mode analysis has been extensively conducted both theoretically and numerically. Due to increasing computing power, it has become possible to determine, numerically, the stationary (unstable) solutions of the Navier-Stokes equations in various flow geometries (Daube and Le Quéré 2002; Gallaire et al. 2006; Brès and Colonius 2008; Ehrenstein and Gallaire 2008; Alizard and Robinet 2008; Marquet et al. 2009), and to investigate the linear stability properties of the basic state. Linear stability analysis provides frequencies and growth rates of the modes against which the basic solution becomes unstable. Global modes are thus obtained by using a linearised version of the flow dynamics equations. In fluid mechanics, however, the equations governing the state evolution are nonlinear. Therefore, a generalisation of modal decomposition would consist in defining an evolution operator which catches the nonlinear features of the flow. This is achieved by the dynamic mode decomposition proposed by Schmid and Sesterhenn (2008). The evolution operator is also defined as a Koopman operator in Rowley et al. (2009), which acts on one flow realisation to generate the next one. According to Rowley et al. (2009), when the dynamics is restricted to an attractor, the Koopman modes may be calculated by harmonic averages, which for finite-time datasets reduce to discrete Fourier transforms (DFT). 
Such a description, therefore, requires time-resolved information over the entire spatial field. This is imposed by the fact that both temporal and spatial relative phases are constitutive of the coherent structures. Nowadays, such a goal can be reached, experimentally, by the use of time-resolved particle image velocimetry (TR-PIV). It produces both temporally and spatially resolved velocity fields of the flow. Acquisition rates can be up to several $\mathrm{kHz}$, which is high-enough for many self-sustained oscillating flows. In this paper, relevant conditions are gathered to access experimentally to the modal structures of an incompressible open-cavity flow, for which several global-mode analyses have been numerically conducted (Brès and Colonius 2008; Dergham et al. 2008; Marquet et al. 2009).

Cavity flows involve shear layers, unstable with respect to Kelvin-Helmholtz waves. In the case of free shear layers, a wide and continuous range of spatial disturbances is amplified, while advecting downstream. Power spectra, for such flows, are usually widely distributed around a most unstable frequency whose value can be predicted theoretically (Huerre and Rossi 1998). On the contrary, when they are externally driven, shear layers behave like amplifiers, promoting the enhancement of the forcing frequencies and their nonlinear interaction, as was experimentally shown by Miksad in the early 1970s (Miksad 1973). In addition, depending on the flow velocity with respect to the KelvinHelmholtz wave celerity, the shear layer instability may change from convective to absolute, giving rise to some global instability (Huerre and Monkevitz 1985).

When a shear layer is impinging on an edge, such as a cavity trailing corner, strong self-sustained oscillations occur. Through pressure, information at impingement is fed back upstream. The resulting feedback-loop enhances self-sustained waves and imposes a global phase coherence to the flow. Depending on cavity length, resonances can be triggered, which give rise to harmonic families of hydrodynamic modes or monochromatic acoustic radiations (tones) (see Rockwell and Naudascher 1979). Consequently, power spectra, calculated from velocity (or pressure) signals, are in the form of well-defined frequency distributions and usually exhibit only a few peaks. The dominant frequency is common to the entire flow, which is typical of a global flow organisation. In incompressible flows, the information is instantaneously fed back to the upstream flow, the sound velocity being much larger than any characteristic velocity of the flow. In addition, a second loop can take place through the insidecavity flow recirculation. In this paper, spatial structures associated with any frequency, in an incompressible opencavity flow, are identified by using discrete Fouriertransforms on time-resolved velocity fields obtained by high-speed PIV.

\section{Experimental setup and basic flow properties}

\subsection{Wind-tunnel}

The study is carried out in an open wind-tunnel described in Fig. 1. The experimental facility is composed of a centrifugal fan providing stationary volume flow upstream of the wind-tunnel, a settling chamber that contributes to equalise the flow and a honey-comb panel, placed at the inlet of the contraction, which contributes to laminarise the flow. In the measurement region, the boundary layer develops above a $300 \mathrm{~mm}$-long plate with profiled leading edge. Wind-tunnel span is equal to $300 \mathrm{~mm}$. Distance between top and bottom walls is $75 \mathrm{~mm}$, large enough to avoid cavity flow perturbation due to the top boundary layer. Complete optical access is made available using reflection-treated glass walls for the wind-tunnel as well as for the cavity setup. Velocities used in the present experiment range from $0.9 \mathrm{~m} / \mathrm{s}$ to $4.2 \mathrm{~m} / \mathrm{s}$. At such very low Mach numbers, the flow can be considered as incompressible. Besides, at the frequencies of the flow, acoustic modes have wavelengths $\lambda_{c}$ much larger than the cavity length $L$. Aero-acoustic feedback mechanism is therefore absent from the flow and the pressure-based feedback mechanism can be seen as instantaneous (Rockwell and Naudascher 1979). Vein noise has been estimated based on laser Doppler velocimetry measurements. Background turbulence is less than $1 \%$. The wind tunnel forces frequencies mainly distributed from 0 to $10 \mathrm{~Hz}$, with a maximal power around $1 \mathrm{~Hz}$ of about $-60 \mathrm{~dB}^{1}$.

\subsection{Time-resolved particle image velocimetry}

TR-PIV requires cameras and lasers with a high repetition rate. Light source is a dual-head New Wave PEGASUS laser. Each laser head supplies a maximal energy of $10 \mathrm{~mJ}$ at repetition rate of $1 \mathrm{kHz}$, at wavelength $527 \mathrm{~nm}$. Each pulse lasts $180 \mathrm{~ns}$. In order to get maximal brightness, both laser sources are used simultaneously, which implies a time sampling $\delta t$ fully dependent on the camera repetition rate. Seeding particles are liquid droplets of mineral oil DEHS - di(2-ethylhexyl)sebacate-, sprayed at the fan entrance. DEHS density is 0.9 and droplet diameters are of the order of $1 \mu \mathrm{m}$. Measurements only start after the seeding particle distribution is uniform inside the cavity ${ }^{2}$. The camera is a Photron FASTCAM-APX RS, whose full resolution is $1,024 \times 1,024$ pixels, at repetition rates up to $3 \mathrm{kHz}$.

\footnotetext{
${ }^{1}$ In comparison, for the same frequency range in the cavity spectrum, power is of the order of $-30 \mathrm{~dB}$, i.e. three orders of magnitude larger.

2 On the contrary, for visualisation, spectacle smoke (liquid droplets of glycerol) seeds the flow; smoke must not be uniformly distributed within the cavity, since optical contrast is used to reveal coherent structures.
} 
Fig. 1 Scheme of the experimental setup, a windtunnel, b cavity sub-system with $L / H=2.0$ (a)

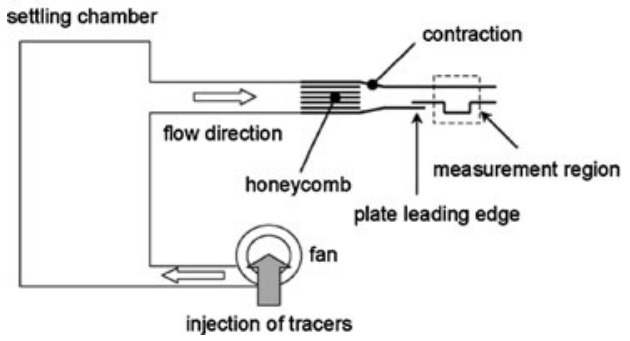

(b)

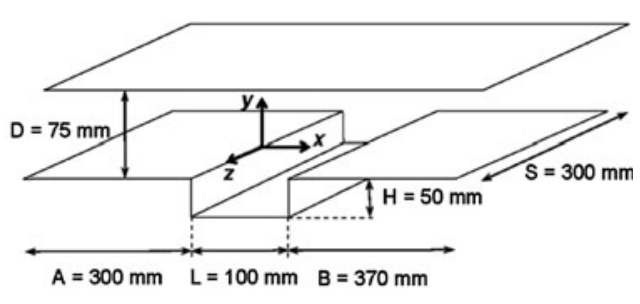

Images are encoded over 8 bits. At such high repetition rates, memory needs to be drained off towards an external hard drive before starting another frame series. Camera onboard memory is $2.6 \mathrm{~GB}$, filled up in about $2.5 \mathrm{~s}$ at full resolution and repetition rate of $1 \mathrm{kHz}$. The flow highest frequency imposes the minimal sampling frequency, for preventing spectral aliasing, according to the ShannonNyquist criterion. Flow characteristic frequencies (including their harmonics) are already known as up to about $100 \mathrm{~Hz}$ for the investigated cases, imposing sampling frequencies larger than $200 \mathrm{~Hz}$. Camera repetition rate is set to $f_{\text {piv }}=500 \mathrm{~Hz}$. In order to save computational-time, velocity fields are sampled at $f_{\text {field }}=f_{\text {piv }} / 2=250 \mathrm{~Hz}$, which satisfies the Shannon criterion. In regard to the cavity flow, we are also interested in very low frequencies. The lowest resolved frequency, which is also the frequency step, depends on the recording time length. Because overlap-averaging is used, getting down to $0.1 \mathrm{~Hz}$ requires a recording time of $20 \mathrm{~s}$. In order to get longer records, image resolution has to be reduced. Consequently, depending on whether the shape ratio $L / H$ is 2 or 1.5 , spatial resolution is lowered to $640 \times 400$ or $512 \times 400$. In both cases, images cover an area $0 \leq x / L \leq 1.1$ by $-1 \leq y / H \leq 0.4$, with a magnification of $0.176 \mathrm{~mm} /$ pixel. Corresponding records last from $16 \mathrm{~s}$ to $21 \mathrm{~s}$, consisting of 8,192 to 10,484 successive images, which corresponds to 4,096 to 5,242 successive displacement fields, respectively, depending on the experimental configuration. Still, such recording durations are too small in regard to very low frequencies. Therefore, in order to preserve reasonable statistics on events occurring at such frequencies, longer recording-times are required, which is achieved by increasing the number of records. That is why fifteen records are performed.

The PIV measurement plane is set at $z / S=0.07$, instead of the usual median plane at $z / S=0$, in order to avoid symmetry planes of the flow. Displacement fields are computed using an optical flow algorithm which relies on an orthogonal dynamical programming (Quénot 1992; Quénot et al. 1998). This algorithm, based on the minimisation of optical intensity difference between two images of particles, has been shown to be one of the most efficient in shear layers, boundary layers or more generally in strong gradient area with respect to other algorithms (Quénot et al. 2001; Stanislas et al. 2005). In a hierarchical processing scheme, the global image-to-image transformation is found by minimising the Minkowski distance between both images. The minimisation procedure is applied iteratively on horizontal stripes of decreasing height, then on vertical stripes of decreasing width. It ends up with a relative displacement field defined at each pixel of the grid. The displacement field is mapped into a velocity field using a calibrated image (rule). The time step between two images, $\delta t=2 \mathrm{~ms}$, has been optimised for the inside and shear flow particle displacements, where false vectors are therefore in a few amounts, essentially occurring in the vicinity of light reflexions. On the contrary, the number of false vectors is larger in the faster quasi-parallel flow outside of the cavity, where particle displacements are at least ten times larger. However, since the outflow remains roughly two dimensional (small transverse velocity component), outflow seeding particles remain in the measurement plane, during $\delta t$, if the plane is carefully aligned with the external flow. Longer $\delta t$ can thus be undertaken, such as to guarantee enough precision for the inside-flow velocity field. When calculation fails (exploded fields or large area of false vectors), three successive particle images, instead of two, are used, which helps in stabilizing the minimisation process, though smoothing the displacement field. At last, it remains less than $1 \%$ of outliers. Searching for outliers is based on the temporal behaviour of the displacement vector at one given point in space. They are detected when the displacement vector is beyond either one threshold value: (i) a threshold value defined relatively to the mean displacement, at the same spatial point, over a finite-time slipping window, (ii) a maximal slope allowed in the rate of change of the displacement vector, relatively to the time-series standard deviation. When detected, outliers are removed by linear interpolation between the previous (past) and next (future) displacement values, at the spatial point under consideration. The procedure is then pursued iteratively until no outlier be no more detected.

Computation time for one field, cropped over a subdomain of $108.1 \times 70.5 \mathrm{~mm}(613 \times 400$ pixels $)$, is of the order of $100 \mathrm{~s}$, on a $3.7 \mathrm{GHz}$ PC processor. When each 
record is made of 8192 frames, providing 4,096 velocity fields, the overall calculation time represents about 110 hours. TR-PIV implying large amount of data makes it impossible to compute the Fourier analysis over the entire field. Instead, spatial subdomains are defined. Lower resolution meshes, getting 1 pixels over 7 in both directions, are defined for global maps. A subdomain at full resolution can also be considered for refinement in the impinging shear layer. Both meshes are illustrated in Fig. 7.

\subsection{Laser Doppler velocimetry}

Laser Doppler velocimetry (LDV) measurements are performed at the very same conditions as the PIV measurements. A continuous argon-ion laser, with a wavelength of $488 \mathrm{~nm}$, is used. Several temporal LDV records are acquired before and after PIV. They are performed on component $V_{x}$, at point $\boldsymbol{r}_{l d v}$ set $5 \mathrm{~mm}$ above and $5 \mathrm{~mm}$ upstream of the cavity trailing edge (see Fig. 7). At the LDV point, shear layer oscillations are of highest amplitude and the low frequencies, resulting from interaction with the trailing corner, are powerful as well. LDV can provide long records (typically 5 minutes), which is useful for compensating TR-PIV limitations in length of time-series. LDV count rates are of the order of $2 \mathrm{kHz}$. Time-series are then equally resampled at the mean particle-sampling frequency by linear interpolation before performing Fourier analysis.

\subsection{Cavity flow description}

Cavity span, $S=30 \mathrm{~cm}$, is given by the spanwise dimension of the wind-tunnel. Cavity height is fixed to $H=50 \mathrm{~mm}$. Span to height ratio is $S / H=6$, which allows the development of a fully three-dimensional (3D) flow, as reported in Faure et al. $(2007,2009)$. Such 3D organisation is believed to significantly contribute to the low-frequency band in the Fourier spectrum. A cartesian coordinate system $\left(\boldsymbol{e}_{x}, \boldsymbol{e}_{y}, \boldsymbol{e}_{z}\right)$ is set mid-span at the cavity leading corner. Various cases of streamwise shape ratio $L / H$ have been undertaken, by modifying the cavity length $L$. Experimental campaign concerns upstream flow Reynolds numbers $R e_{L}=U_{\infty} L / v$ from 5,700 to $13,500 . U_{\infty}$ is defined as the velocity outside of the boundary layer, at the cavity leading edge $(x=0)$. In all cases, the shear layer experiences self-sustained oscillations. Driven by the outflow, the insideflow is put into motion, generating a main fluid recirculation inside the cavity, from the trailing edge over about the cavity length. For some shape ratio, a secondary, smaller, counter-rotating vortex forms upstream of the main-vortex, close to the cavity leading edge. At impinging corner, outflow is periodically injected inside the cavity, at the frequency of the shear layer oscillations, and advected back to the shear layer via the main-vortex, as displayed in Fig. 2 for two configurations. This mechanism provides a secondary delayed feedback mechanism for the flow. A representative instantaneous velocity field is shown in Fig. 3 for the case $L / H=2.0, R e_{L}=12,180\left(U_{\infty}=1.90 \mathrm{~m} / \mathrm{s}\right)$, together with the corresponding vorticity field $\omega_{z}$. The shear layer interacts with the trailing corner that generates an impinging vortex. In time, mass fluxes are cyclically exchanging fluid between the inside and outside flows, due to shear layer oscillations. Nonlinear effects strongly influence the impinging region, resulting into amplitude and frequency modulations (Knisely and Rockwell 1982).

The velocity field can be decomposed into a mean velocity field $\left(\bar{V}_{x}, \bar{V}_{y}\right)$ and a velocity fluctuation field $\left(u^{\prime}, v^{\prime}\right)$, such as $V_{x}=\bar{V}_{x}+u^{\prime}$ and $V_{y}=\bar{V}_{y}+v^{\prime}$. The mean flow field modulus, $\bar{U}(\boldsymbol{r})=\sqrt{\bar{V}_{x}^{2}+\bar{V}_{y}^{2}}$ and $\bar{V}_{x}$ profiles, at five streamwise positions, are shown in Fig. $4 \mathrm{a}$, for the configuration $L / H=2, R e_{L}=12,180$. The boundary layer displacement thickness $\delta_{1}=2.956 \mathrm{~mm}$ and momentum thickness $\delta_{2}=1.294 \mathrm{~mm}$, are calculated from $\bar{V}_{x}(y)$ at the leading edge of the cavity $(x=0)$. The shape factor $H=\delta_{1} / \delta_{2}$ is therefore equal to 2.28 , indicating that the boundary layer is not perfectly laminar. However, the $L_{2}$-norm of the difference between the experimental profile and its Blasius fit is less than $1 \%$. From the Blasius fit, the boundary layer thickness is estimated at $\delta_{0}=7.83 \mathrm{~mm}$. Elements $\overline{u^{\prime} v^{\prime}}, \overline{u^{\prime 2}}$ and $\overline{v^{\prime 2}}$, of the Reynolds tensor are shown in Fig. 4b, c, d. If one considers independently time-series of 4,096 field realisations each, statistical convergence is already within $2 \%$ for the mean flow and within $10 \%$ for Reynolds tensor components. Furthermore, convergence is increased by averaging over 15 different recordings. As expected, the Reynolds tensor components, $\overline{u^{\prime 2}}, \overline{v^{\prime 2}}, \overline{u^{\prime} v^{\prime}}$, clearly show that the fluctuation energy is concentrated in the impinging shear layer, near the cavity trailing edge. Indeed, the shear layer flapping motion is the most energetic phenomenon in the flow. In addition, intensity distribution of velocity fluctuations is not equivalent for $x$ and $y$ components. In particular, $\overline{u^{2}}$ (Fig. 4c) exhibits a structure in two lobes distributed on both sides of the cavity top-plane $(y=0)$, while $\overline{v^{\prime 2}}$ (Fig. 4d) has a gaussian-like shape centred on the same position. Similar organisation was observed by Kuo and Huang (2001), using shear layer transverse profiles in various cavity geometries. The structure in $\overline{u^{\prime} v^{\prime}}$ shows that $u^{\prime}$ and $v^{\prime}$ are, in average, of opposite signs atop, and of the same sign below, the cavity top-plane.

\section{Modal structures}

The 2D-2C time-resolved velocity fields, obtained by TRPIV, provide over-sampled time-series of both $u^{\prime}$ and $v^{\prime}$ at 
(a)

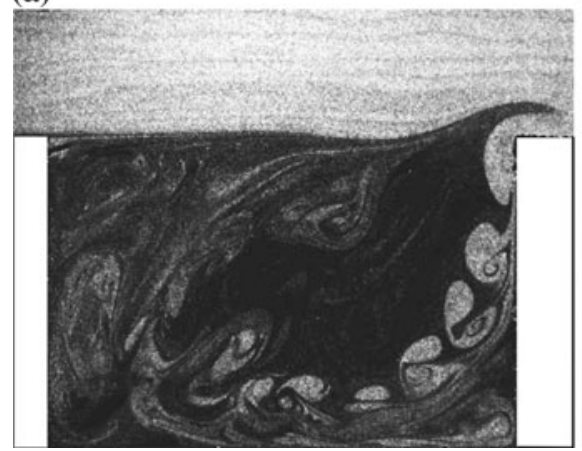

(b)

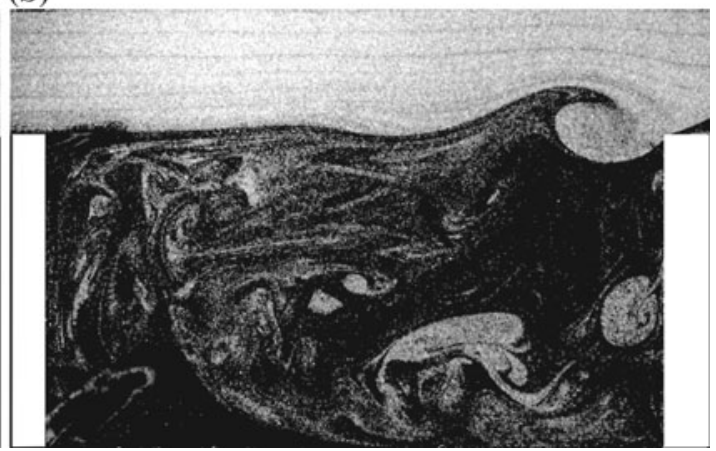

Fig. 2 Smoke-visualisation of cavity flow for two configurations. a With an upstream velocity $U_{\infty}=2.31 \mathrm{~m} / \mathrm{s}\left(R e_{L}=11,150\right)$ and an aspect ratio $L / H=1.5$. b With an upstream velocity $U_{\infty}=1.90 \mathrm{~m} / \mathrm{s}\left(R e_{L}=12,180\right)$ and an aspect ratio $L / H=2.0$

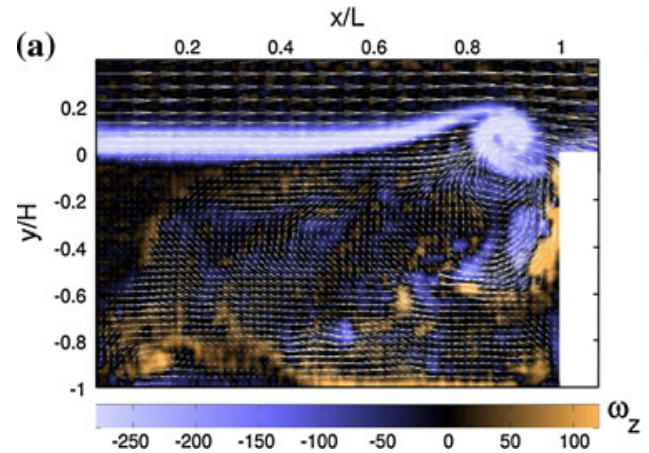

Fig. 3 Instantaneous 2D-2C velocity field (arrows) together with vorticity component $\omega_{z}$ (colormap), for $\operatorname{Re}_{L}=12,180\left(U_{\infty}=1.90\right.$ $\mathrm{m} / \mathrm{s}$ ), $L / H=2.0$ : a raw total velocity field, $\mathbf{b}$ fluctuation field (mean

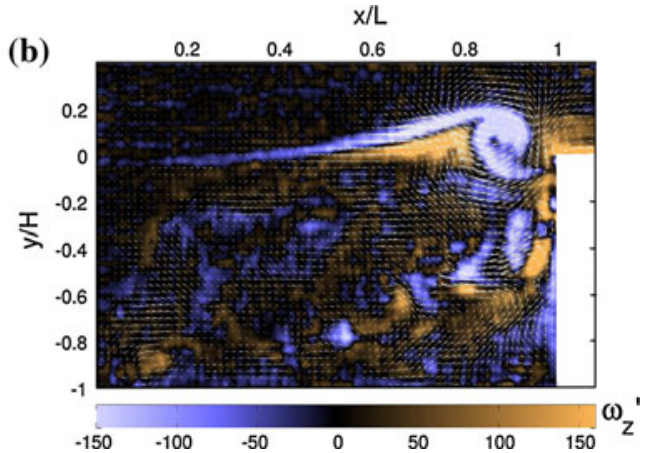

flow substraction). Color scales have been truncated in order to ease features observation. a Total vorticity $-428 \mathrm{~s}^{-1} \leq \omega_{z} \leq 330 \mathrm{~s}^{-1}$, b fluctuating vorticity $-298 \mathrm{~s}^{-1} \leq \omega_{z}^{\prime} \leq 350 \mathrm{~s}^{-1}$
Fig. 4 Statistics performed over $4,096 \times 15$ velocity fields from 15 different records, in the case $R e_{L}=12,180\left(U_{\infty}=1.90\right.$ $\mathrm{m} / \mathrm{s}) ; L / H=2$. a Mean flow field $\bar{V}_{x}, \bar{V}_{y}$ (arrows) and modulus $\bar{U}$ (color scale) ; intensity of velocity field fluctuations: components b $\overline{u^{\prime} v^{\prime}}, \mathbf{c} \overline{u^{\prime 2}}, \mathbf{d} \overline{v^{\prime 2}}\left(\right.$ in $\left.\mathrm{m}^{2} / \mathrm{s}^{2}\right)$
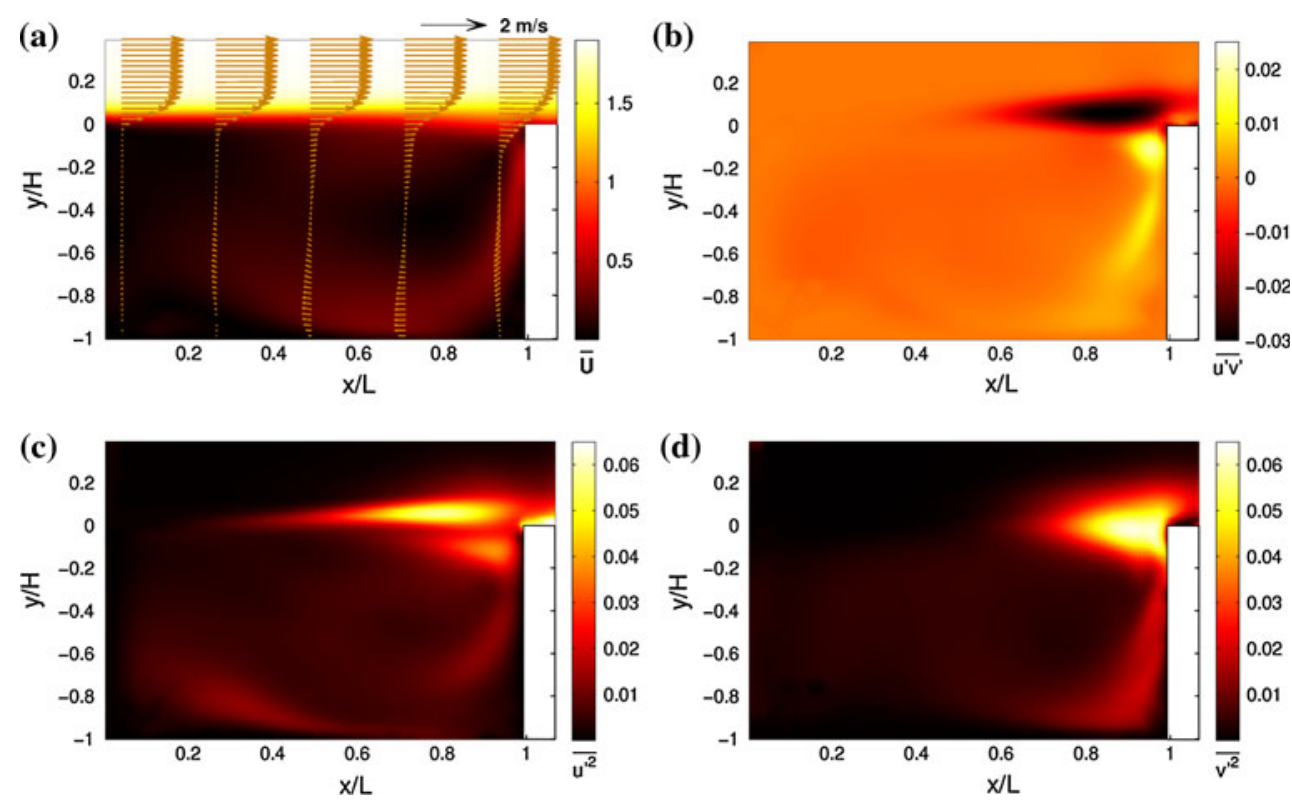

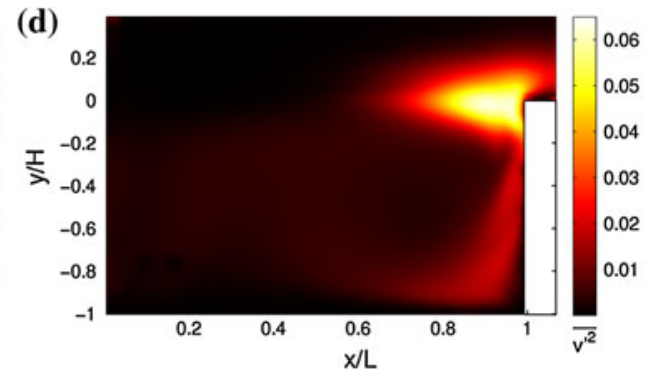

each point of the spatial grid. From these data sets, spatial structures can be obtained; results are discussed in Sect. 3.3. Before investigating in more details these modal spatial structures, it may be instructive to first discribe the spectral signature of the flow at some representative location. 
3.1 Power spectral density at the LDV point and TRPIV validation

LDV measurements are conducted as a reference metrology. Both TR-PIV and LDV power spectral densities (PSD), from the LDV point $\left(\boldsymbol{r}_{l d v}\right)$, are shown in Fig. 5, for three different cases. Each TR-PIV power spectral density has been obtained by averaging over the 15 independent PIV records performed in the very same conditions. PSDs are obtained with the help of a Welch algorithm using a Hamming window function $h(t)$, which writes, in the discretised form

$h_{\mathrm{j}}=0.54-0.46 \cos \left(\frac{2 \pi j}{n-1}\right)$

Window-size is chosen equal to about $9 \mathrm{~s}$, with an overlap of $98 \%$, such as the frequency step $\delta f$ is about $0.1 \mathrm{~Hz}$ for both LDV and TR-PIV spectra. Significant peaks in spectra are typically broader than $1 \mathrm{~Hz}$. For the $\xi^{\text {th }}$ window, PSD is defined as

$W_{\mathrm{k}}^{\xi}=\operatorname{DFT}\left\{u^{\prime \xi} h^{\xi}\right\}=\sum_{\mathrm{j}=(\xi-1) n}^{\xi n-1} u_{\mathrm{j}}^{\prime} h_{\mathrm{j}} e^{-\frac{2 i \pi}{n} \mathrm{jk}}$

with $t=j \delta t$ and $f=k \delta f=k /(n \delta t)$. One LDV recording provides $N_{w d w}^{l d v}=1,500$ windows for averaging, while TRPIV time-series each consists of 50 windows. However, since 15 independent TR-PIV records are used, power spectra obtained from TR-PIV velocity fields are actually averaged over $N_{w d w}^{p i v}=750$ windows. With these parameters, $95 \%$ confidence intervals are equal to $[-0.280 \mathrm{~dB}+$

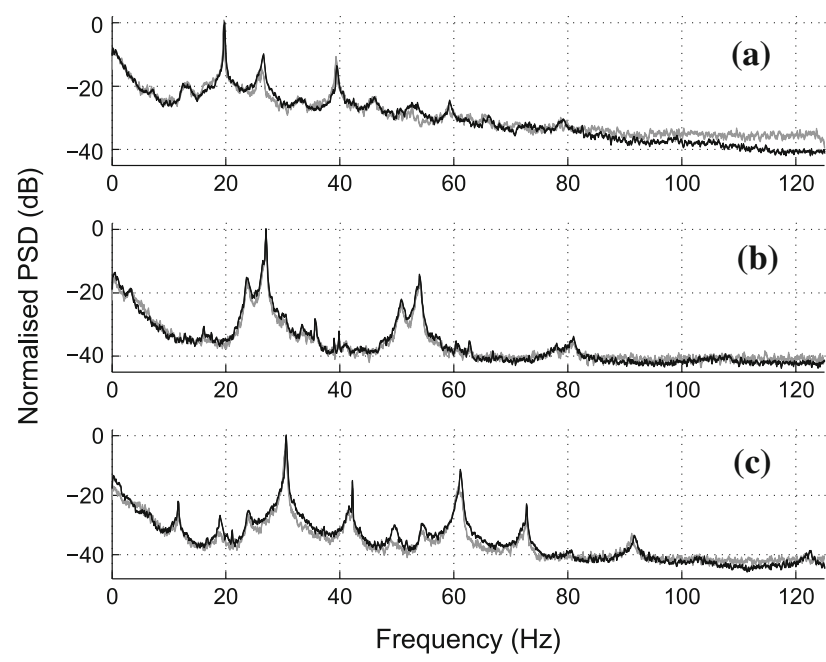

Fig. 5 Power spectral density, at the LDV point, normalised by the maximal value of LDV spectrum; for a $L / H=2.0, R_{L}=12,180$ $\left(U_{\infty}=1.90 \mathrm{~m} / \mathrm{s}\right) ; \quad$ b $L / H=1.5, \quad R e_{L}=10,050\left(U_{\infty}=2.09 \mathrm{~m} / \mathrm{s}\right) ;$ c $L / H=1.5, \operatorname{Re}_{L}=11,150\left(U_{\infty}=2.31 \mathrm{~m} / \mathrm{s}\right)$. PSD from the LDV time-series (dark line) and TR-PIV velocity fields (grey line). The line thickness represents the $95 \%$ confidence interval
$0.288 \mathrm{~dB}$ ] for LDV and [ $-0.393 \mathrm{~dB}+0.409 \mathrm{~dB}$ ] for TRPIV spectra, respectively. The analytical expression of Fourier spectra averaging is further given by

$\bar{W}_{\mathrm{k}}=\frac{1}{N_{w d w}} \sum_{\xi=1}^{N_{w d w}} W_{\mathrm{k}}{ }^{\xi}$.

Power spectral densities are normalised and expressed in $\mathrm{dB}$ units according to

$w_{\mathrm{k}}=10 \cdot \log _{10}\left(\frac{\bar{W}_{\mathrm{k}}}{\max \left(\bar{W}_{\mathrm{k}}\right)}\right)$.

Depending on the case, agreement between LDV and TR-PIV results is within $4 \times 10^{-3}$ to $4 \times 10^{-2}$ for the mean velocity at the LDV point, the largest scattering being explained by some evolution in the experimental parameters, mainly due to temperature variations. The same is true for the frequency matching. Contrary to spectrum at $L / H=1.5, R e_{L}=10,050$, where both LDV and TR-PIV spectral amplitudes are in good agreement at high frequency (Fig. 5b), it can be seen, at $L / H=2$ and $R e_{L}=12,180$, that amplitudes saturate at a constant level, in TR-PIV, for frequencies greater than 100, while the energy cascade still goes on in the LDV spectrum (Fig. 5a). Energy cascade range is broader at $L / H=2$ and extents down to $120 \mathrm{~Hz}$, because mixing is more efficient in the shear layer for larger $L$. The third harmonic $4 f_{a}=122 \mathrm{~Hz}$, for the configuration $L / H=1.5, R e_{L}=11,150$, cannot be clearly observed in the TR-PIV spectrum (Fig. 5c). It is caused by an overlap of higher frequencies due to a lower Nyquist frequency in TR-PIV time-series. TR-PIV data, for cases (a) and (c), are therefore marginally sufficiently sampled. Nevertheless, TR-PIV proves to give greatly satisfying results for spectral analysis, in regard to frequencies and amplitudes.

\subsection{Spectral data description}

Featuring the cavity flow, PSDs exhibit in all three cases a dominant frequency, $f_{a}$, along with its first two or three harmonics $2 f_{a}, 3 f_{a}$ and $4 f_{a}$, as well as various secondary peaks. For the sake of illustration, we will focus on the case $L / H=2.0, R e_{L}=12,180$, because the spectral distribution exhibits several characteristics which are common to all spectra in the present experiment and more generally, are typical of cavity oscillations in incompressible flows as described in earlier studies (Knisely and Rockwell 1982; Rockwell 1983). The same analysis can be done parametrically by varying $L / H$ and $U_{\infty}$.

Power spectral densities, issued of TR-PIV time-series, can be considered at various locations, in the field, for both velocity fluctuation components $u^{\prime}(\boldsymbol{r}, t)$ and $v^{\prime}(\boldsymbol{r}, t)$ since we are dealing with two-components-space-extended data. 
For the sake of clarity, power spectral densities will be noted as functions of frequency $f$, though being discrete objects,

$\bar{W}_{\mathrm{k}} \longrightarrow \bar{W}_{x, y}(\boldsymbol{r}, f)$.

Space-extended PSDs are normalised and expressed in $\mathrm{dB}$ units as before, in the case of single time-series, according to

$w_{x, y}(\boldsymbol{r}, f)=10 \cdot \log _{10}\left(\frac{\bar{W}_{x, y}(\boldsymbol{r}, f)}{\max _{\boldsymbol{r}, f}\left(\bar{W}_{x, y}(\boldsymbol{r}, f)\right)}\right)$,

such that PSD are normalised, among all frequencies and over any spatial point, with respect to the highest value of the dominant frequency. Consequently, $w_{x, y}(\boldsymbol{r}, f) \leq 0$ is equal to 0 where $\bar{W}_{x, y}\left(\boldsymbol{r}, f_{a}\right)$ is maximum.

Figure 6 illustrates, in more details, the general structure of the spectrum. It shows PSDs of both $u^{\prime}$ and $v^{\prime}$, picked up at a given point in the flow $\boldsymbol{r}_{p}=(0.85 \mathrm{~L}, 0.06 \mathrm{H})$. A predominant frequency and some of its harmonics are clearly detected $\left(f_{a}=19.7 \mathrm{~Hz}, 2 f_{a}=39.3 \mathrm{~Hz}, 3 f_{a}=59.1 \mathrm{~Hz}\right.$ and $4 f_{a} \simeq 78.8 \mathrm{~Hz}$ ). The Strouhal number associated with the predominant mode, $S t_{a}=f_{a} \delta_{2} / U_{\infty}=0.014$-based on the incoming boundary layer momentum thickness $\delta_{2} \simeq 1.3 \mathrm{~mm}$ estimated at the leading edge of the cavity $(x=0)$ - , is relatively close to the value predicted from a linear stability analysis of a hyperbolic tangent-like free shear layer, $S t_{c}=0.017$, and in good agreement with reported values in literature (Michalke 1964; Ziada and Rockwell 1982). For instance, Chang et al. (2006) found a Strouhal number of about 0.015 for an incompressible turbulent flow past a rectangular shallow cavity with $L / H=2$.

Multiple additional —right and left-peaks can also be observed along with the $f_{a}$ harmonic family, as mentioned in several previous investigations (Knisely and Rockwell 1982; Lin and Rockwell 2001; Rockwell et al. 2003; Delprat 2006, 2010). They have been denoted $f_{+}=$ $26.3 \mathrm{~Hz}$ and $f_{-}=12.6 \mathrm{~Hz}$ when symmetrically distributed

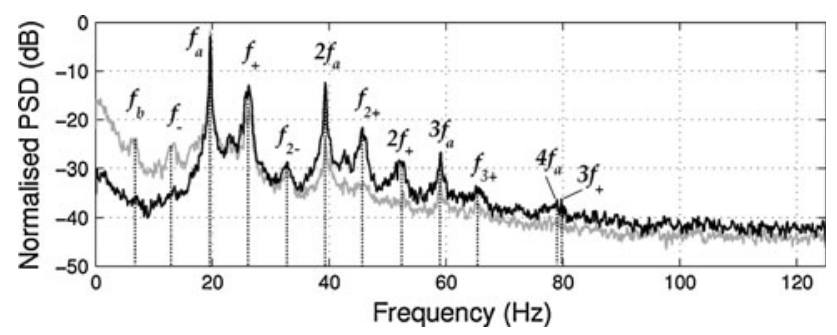

Fig. 6 Normalised power spectral density, in $\mathrm{dB}$, for both velocity fluctuation components $u^{\prime}$ et $v^{\prime}: w_{x}\left(\boldsymbol{r}_{p}, f\right)$ (grey line) and $w_{y}\left(\boldsymbol{r}_{p}, f\right)$ (black line) at one single point of the velocity field, $\boldsymbol{r}_{p}=$ $\left(x_{p}, y_{p}\right)=(0.85 L, 0.06 H)$, for the configuration $L / H=2.0$; $\operatorname{Re}_{L}=12,180\left(U_{\infty}=1.90 \mathrm{~m} / \mathrm{s}\right)$ around $f_{a}, f_{2+}=46.0 \mathrm{~Hz}$ and $f_{2-}=32.6 \mathrm{~Hz}$ around $2 f_{a}$, and $f_{3_{+}}=65.7 \mathrm{~Hz}$ in connection with $3 f_{a}$. Worthy of note is the presence of harmonics $2 f_{+}=52.7 \mathrm{~Hz}$ and $3 f_{+} \simeq$ $79.0 \mathrm{~Hz}$ of $f_{+}$in the case $L / H=2.0, R e_{L}=12,180$, which is not a general feature of the spectra when $L / H$ and $U_{\infty}$ are varied. The appearance of two harmonic families usually corresponds to a mode-switching phenomenon, as reported in Knisely and Rockwell (1982); Kegerise et al. (2004); Pastur et al. (2008). The Strouhal number associated with $f_{+}, S t_{+}=f_{+} \delta_{2} / U_{\infty}=0.018$, is such that $S t_{a}<S t_{c}<S t_{+}$. Therefore, the most dangerous frequency $f_{c}$ would be located between the most two important peaks $f_{a}$ and $f_{+}$.

In addition, a peak at low frequency, $f_{b}=6.6 \mathrm{~Hz}$, is observed (Fig. 6). The existence of such a low frequency, equal to the difference between the dominant frequency and its secondary peaks, is a common feature in most of the various configurations, as it can be seen in Fig. 5. The frequency $f_{b}$ could result from the nonlinear interaction between $f_{a}$ and $f_{+}$, but $f_{+}$could also result from the nonlinear combination of $f_{a}$ and $f_{b}$, via an amplitude modulation at impingment (Knisely and Rockwell 1982; Lucas and Rockwell 1984; Delprat 2006, 2010). Indeed, combinations between the harmonics $n f_{a}$ of $f_{a}$ with $f_{b}$ occur to produce sum frequencies $n f_{a} \pm f_{b}$. For instance, the secondary peak $f_{-}=12.6 \mathrm{~Hz}$ is close to the difference frequency $f_{a}-f_{b}=12.7 \mathrm{~Hz}$. According to this interpretation, and irrespective to the origin of the low-frequency component, global structure of the spectral distribution can be viewed as the result of nonlinear interactions and modulation processes which may combine, as shown in Miksad et al. (1983); Delprat (2006, 2010).

The power spectrum calculated at different spatial points, out of the TR-PIV velocity fields, is depicted in Fig. 7. It can be seen that the main frequency, $f_{a}=19.7 \mathrm{~Hz}$, has the same value everywhere in the flow, while its spectral amplitude actually varies in space. In fact, this feature is common to any peak in the spectrum. It confirms that the flow organisation persists along the entire length of the cavity shear layer and inside the cavity, that is the occurrence of a global organisation, which is a main characteristic of self-sustained oscillations flows (Rockwell and Knisely 1979).

\subsection{Modal structure construction}

Since the initial time is the same for any point of the spatial grid from TR-PIV data, each local velocity fluctuations time-series, at any location $\boldsymbol{r}$, can provide a local complex Fourier-transform given in $(\mathrm{m} / \mathrm{s}) / \mathrm{Hz}$, which is obviously a function of $f=\omega /(2 \pi)$ below the Nyquist frequency $(0 \leq f \leq 125 \mathrm{~Hz})$. In other words, TR-PIV provides the complex spatial structure, $\boldsymbol{S}(\boldsymbol{r}, f)$, associated with any frequency channel, considered in the (global) Fourier 


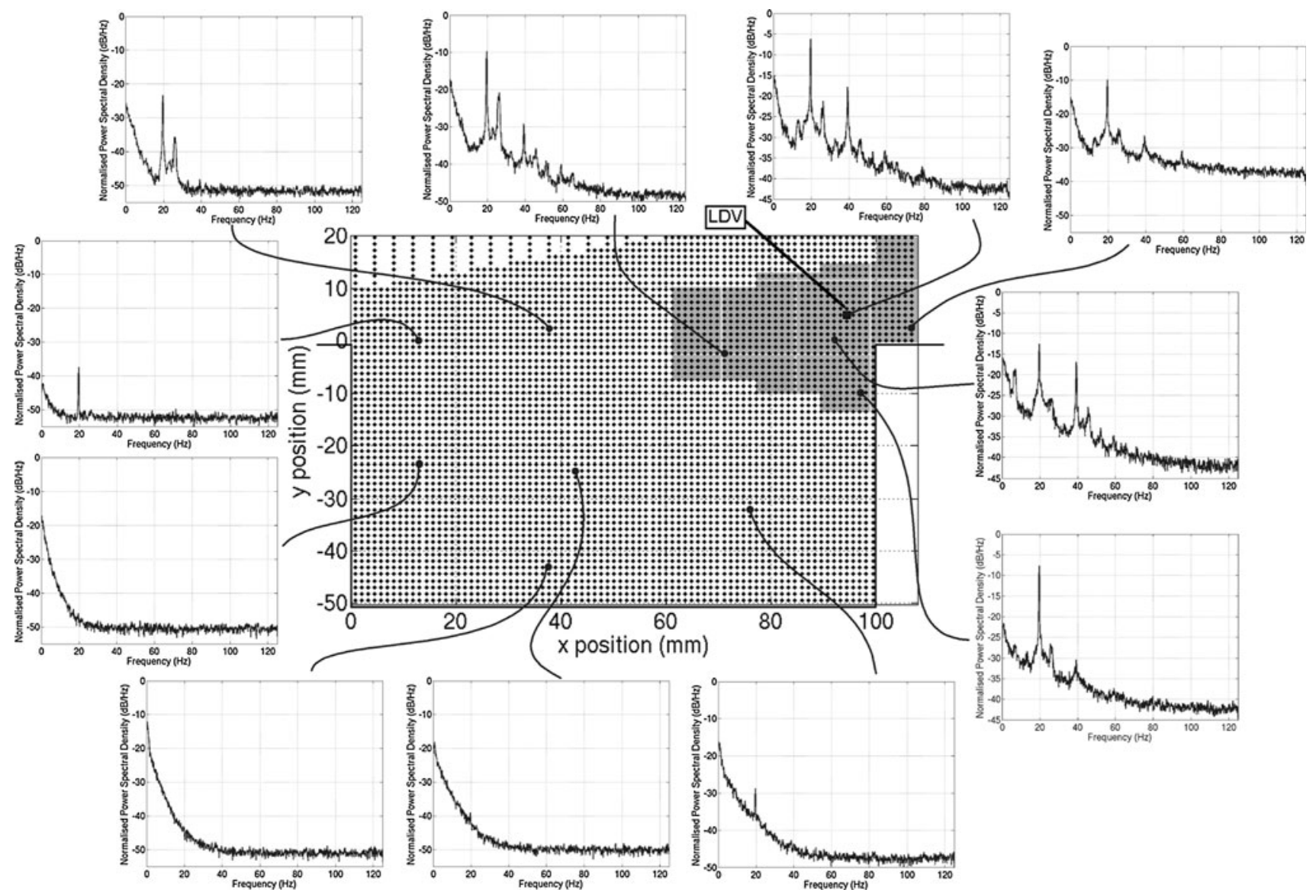

Fig. 7 Subdomains defined for field analyses (in the case $L / H=2.0$ ): low-resolution mesh distributed over space (dots), full resolution in shear layer near the trailing edge (grey region). The

spectrum. $\boldsymbol{S}(\boldsymbol{r}, f)=S_{x}(\boldsymbol{r}, f) \boldsymbol{e}_{x}+S_{y}(\boldsymbol{r}, f) \boldsymbol{e}_{y}$ is calculated on the fluctuating velocity $\boldsymbol{u}^{\prime}=u^{\prime}(\boldsymbol{r}, t) \boldsymbol{e}_{x}+v^{\prime}(\boldsymbol{r}, t) \boldsymbol{e}_{y}$ from a single TR-PIV recording, at point $\boldsymbol{r}$, and is associated with a given frequency channel $f \pm \Delta f / 2$

$\boldsymbol{S}(\boldsymbol{r}, f)=\boldsymbol{S}_{k}(\boldsymbol{r})=\operatorname{DFT}\left\{\boldsymbol{u}^{\prime}(\boldsymbol{r}, j \delta t)\right\}=\sum_{j=0}^{N-1} \boldsymbol{u}^{\prime}(\boldsymbol{r}, j \delta t) \mathrm{e}^{-\frac{2 i \pi j}{N} j k}$,

where $\boldsymbol{u}^{\prime}(\boldsymbol{r}, j \delta t)$ is the velocity fluctuation vector at the $j^{\text {th }}$ time step. These spatial structures are therefore not issued of 2D-spatial-Fourier transforms but maps made of Fourier-transformed spatially distributed time-series. Discrete Fourier-transforms are normalised the same way as PSDs:

$\boldsymbol{s}(\boldsymbol{r}, f)=\frac{\boldsymbol{S}(\boldsymbol{r}, f)}{\max _{\boldsymbol{r}, f}(\boldsymbol{S}(\boldsymbol{r}, f))}$.

We refer to modal spatial structure, or modal structure, when dealing with the spatial distribution of the Discrete Fourier-transforms (DFT) $s_{x, y}(\boldsymbol{r}, f)$, the horizontal and vertical components of $\boldsymbol{S}(\boldsymbol{r}, f)=|\boldsymbol{S}(\boldsymbol{r}, f)| \exp (i \phi(\boldsymbol{r}, f))$
LDV measurement point is shown. Examples of power spectral densities, calculated on the streamwise velocity fluctuating-component $u^{\prime}$, are given at various locations of the velocity field

after normalisation (see Eq. 8). These modal spatial structures are displayed for both components in Figs. 8a, b, 9, 10, 11, 12a, b, 14a, b and 15a, b. PSD maps $w_{x, y}(\boldsymbol{r}, f)$ are shown in Figs. 8c, d, 9, 10, 11, 12, 14, 15c, d. Phase information is lost in PSD, therefore, PSD can be averaged over the 15 available recordings, contrary to DFT. PSD maps are expressed in $\mathrm{dB}$ units, as defined in Eq. (2)-(6).

The most energetic modal structure (at $f_{a}=19.7 \mathrm{~Hz}$ ) is shown in Fig. 8 for both $u^{\prime}$ (left column) and $v^{\prime}$ (right column). In Fig. 8a, b are represented the real part of $s_{x}\left(\boldsymbol{r}, f_{a}\right)$ and $s_{y}\left(\boldsymbol{r}, f_{a}\right)$; in Fig. 8c, d the power spectral density distributions $w_{x}\left(\boldsymbol{r}, f_{a}\right)$ and $w_{y}\left(\boldsymbol{r}, f_{a}\right)$. First $\left(2 f_{a}=\right.$ $39.3 \mathrm{~Hz})$ and second $\left(3 f_{a}=59.1 \mathrm{~Hz}\right)$ harmonics are displayed in Figs. 9 and 10, respectively. The spatial structure of the second most dominant frequency, at $f_{+}=26.3 \mathrm{~Hz}$, is shown in Fig. 11, and its first harmonic $2 f_{+}=52.7 \mathrm{~Hz}$ is displayed in Fig. 12. In addition, the right peak $f_{2+}=46.0 \mathrm{~Hz}$ coming with the harmonic $2 f_{a}$ is presented in Fig. 14. The modal structure associated with $f_{b}=6.6 \mathrm{~Hz}$ is shown in Fig. 15. The colormap encoding DFTs real part has been designed such as to highlight transitions from positive to negative values. 
Fig. 8 Spatial structure of the dominant mode $f_{a}=19.7 \mathrm{~Hz}$. Real part of $\mathbf{a} s_{x}\left(\boldsymbol{r}, f_{a}\right)$ and b $s_{y}\left(\boldsymbol{r}, f_{a}\right)$. Power spectral density distributions $\mathbf{c} w_{x}\left(\boldsymbol{r}, f_{a}\right)$ and $\mathbf{d} w_{y}\left(\boldsymbol{r}, f_{a}\right)$
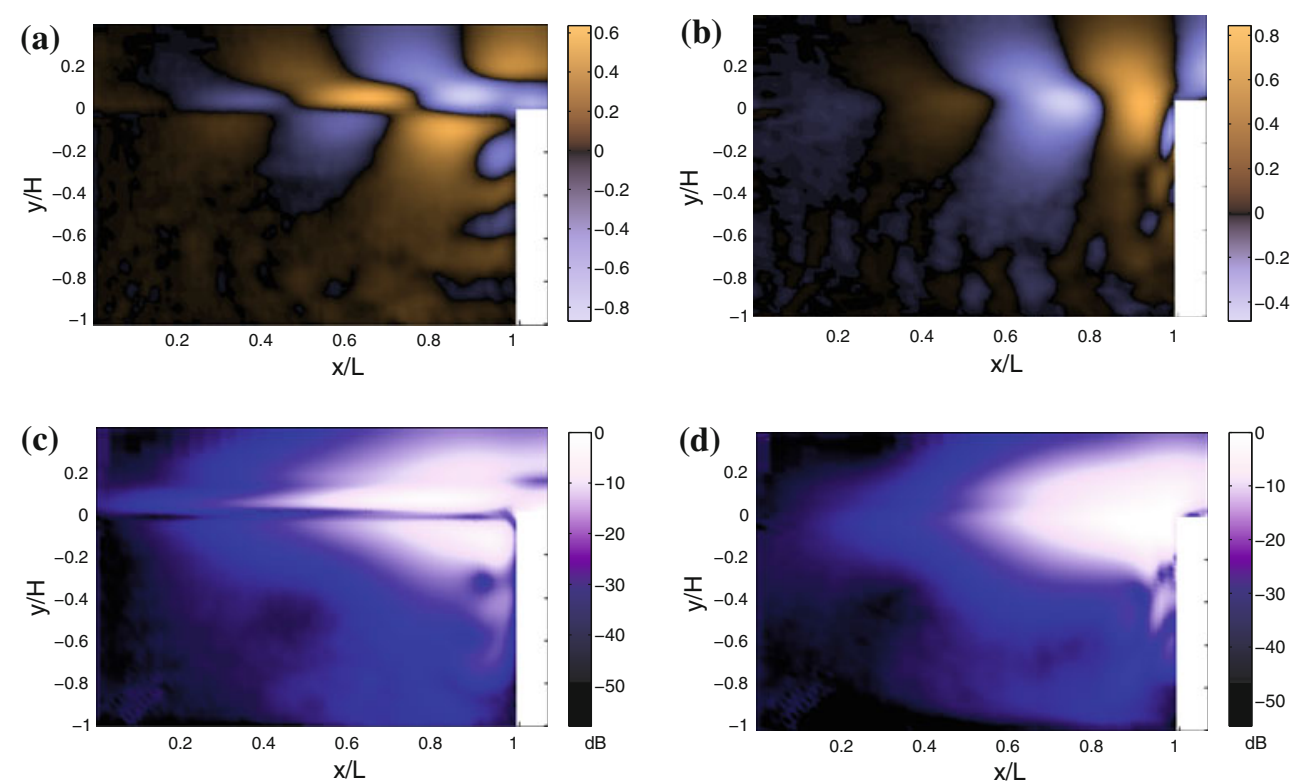

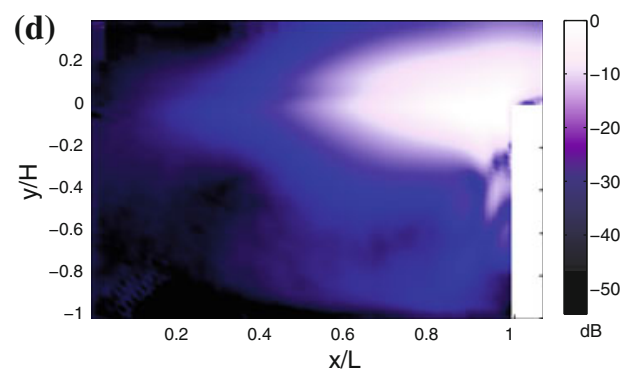

\section{Discussion}

In this Section, we first analyse the spatial distribution associated with the most characteristic temporal frequencies and then discuss the nature of the modal structures in the context of global and Koopman modes.

\subsection{Modal spatial structures and flow features}

- One can remark that $s_{x}$ and $s_{y}$, as well as their PSD $w_{x}$ and $w_{y}$, exhibit very different structures—see Figs. 8, 9, 10, 11, 12, 14, 15a, c and Figs. 8c, d, 9, 10, 11, 12, 14, $15 \mathrm{~b}, \mathrm{~d}$, respectively. The same was already observed for the velocity fluctuation intensities $\overline{u^{\prime 2}}(\boldsymbol{r})$ and $\overline{v^{\prime 2}}(\boldsymbol{r})$ in
Fig. $4 \mathrm{c}$, d. $w_{x}\left(\boldsymbol{r}, f_{a}\right)$ and $w_{y}\left(\boldsymbol{r}, f_{a}\right)$ are moreover very similar to the structure of $\overline{u^{\prime 2}}(\boldsymbol{r})$ and $\overline{v^{\prime 2}}(\boldsymbol{r})$, respectively. Indeed, although fluctuating intensities $\overline{u^{\prime 2}}(\boldsymbol{r})$ and $\overline{v^{\prime 2}}(\boldsymbol{r})$ represent integral values of $w_{x}$ and $w_{y}$ over all frequencies, the amplitude of the dominant frequency is at least one order of magnitude larger than any other peak in the spectrum, making $f_{a}$ also dominant in the fluctuation inte nsities.

- The two harmonic families $f_{a}$ (Figs. 8, 9 and 10) and $f_{+}$ (Figs. 11, 12) correspond to shear layer modes. Their modal structures are powerful in the impinging shear layer, in a vicinity of the cavity trailing edge. The real part of $s_{x}$ or $s_{y}$ exhibit oscillations in the shear layer, of increasing amplitude as getting closer to the trailing
Fig. 9 Modal spatial structure associated with the first harmonic $2 f_{a}=39.3 \mathrm{~Hz}$. Real part of $\mathbf{a} s_{x}\left(\boldsymbol{r}, 2 f_{a}\right)$ and b $s_{y}\left(\boldsymbol{r}, 2 f_{a}\right)$. Power spectral density distributions $\mathbf{c} w_{x}\left(\boldsymbol{r}, 2 f_{a}\right)$ and $\mathbf{d} w_{y}\left(\boldsymbol{r}, 2 f_{a}\right)$
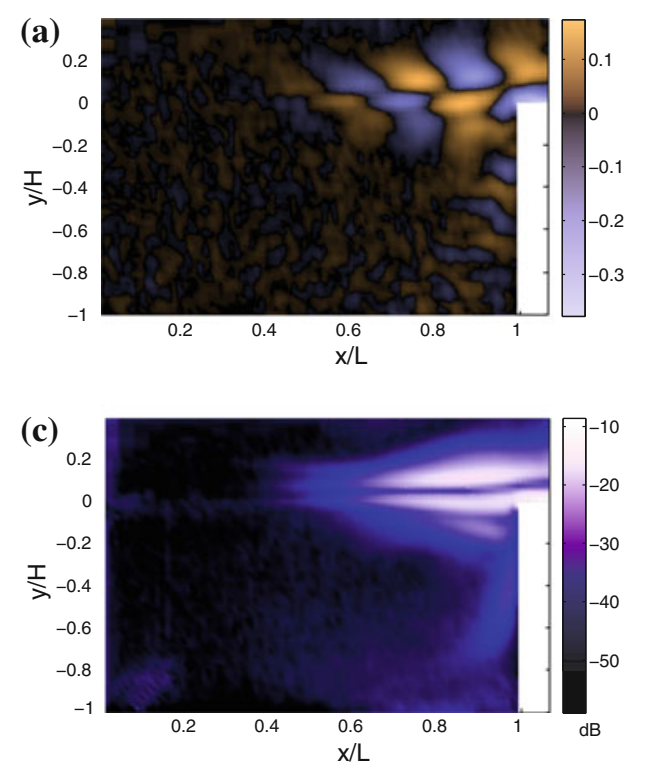
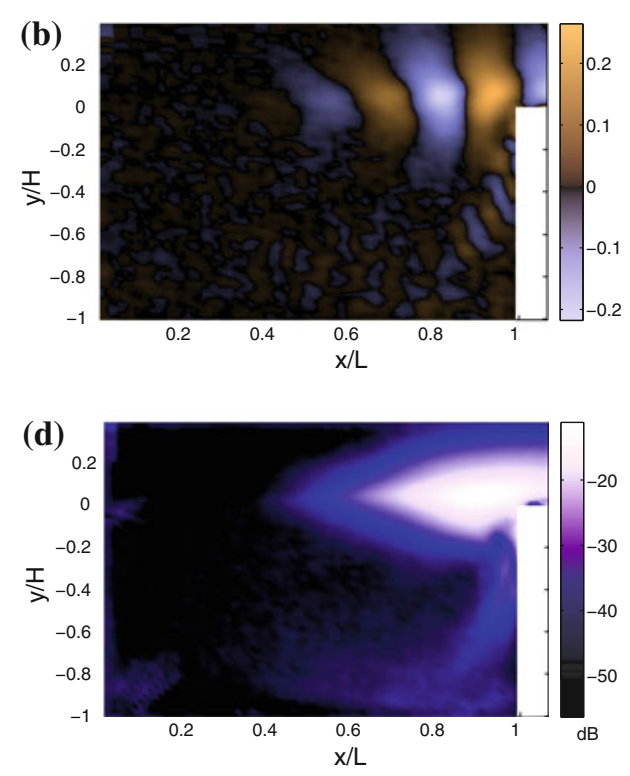
Fig. 10 Modal spatial structure associated with the second harmonic $3 f_{a}=59.1 \mathrm{~Hz}$. Real part of $\mathbf{a} s_{x}\left(\boldsymbol{r}, 3 f_{a}\right)$ and b $s_{y}\left(\boldsymbol{r}, 3 f_{a}\right)$. Power spectral density distributions $\mathbf{c} w_{x}\left(\boldsymbol{r}, 3 f_{a}\right)$ and $\mathbf{d} w_{y}\left(\boldsymbol{r}, 3 f_{a}\right)$
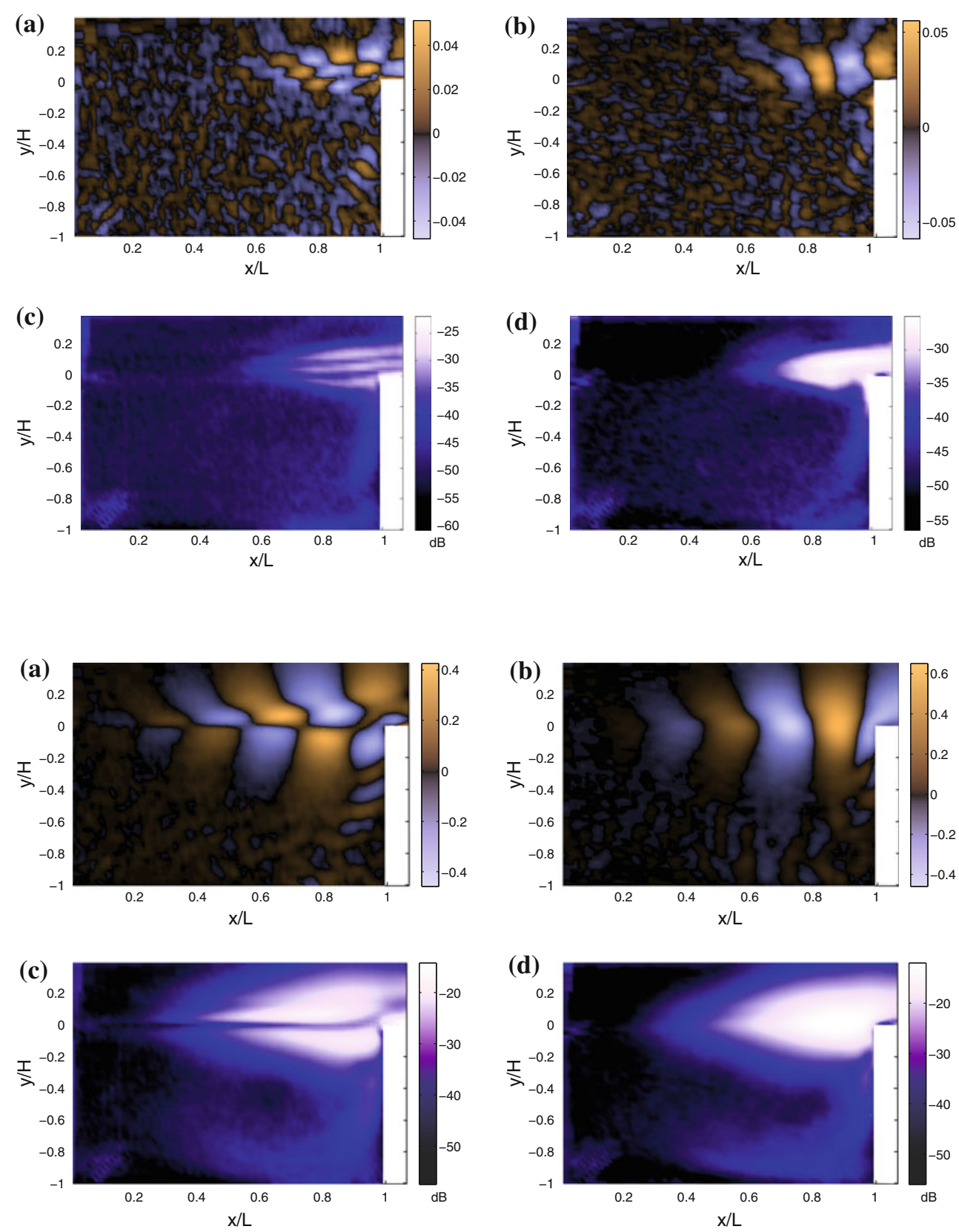

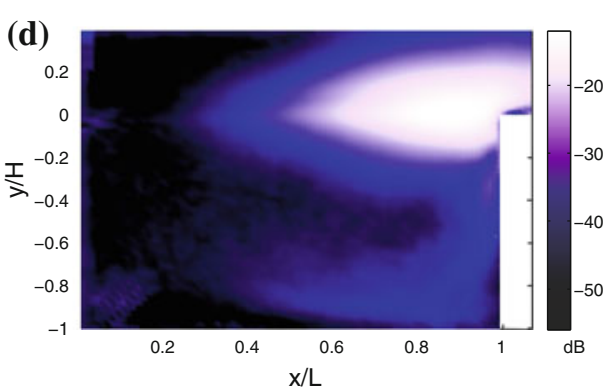

Fig. 11 Spatial structure associated with the second most dominant mode

$f_{+}=f_{a}+f_{b}=26.3 \mathrm{~Hz}$. Real part of $\mathbf{a} s_{x}\left(\boldsymbol{r}, f_{+}\right)$and $\mathbf{b} s_{y}\left(\boldsymbol{r}, f_{+}\right)$. Power spectral density distributions $\mathbf{c} w_{x}\left(\boldsymbol{r}, f_{+}\right)$and d $w_{y}\left(\boldsymbol{r}, f_{+}\right)$ edge, corresponding to an alley of advected counterrotating vortices. Part of the energy of these modes is also present along the downstream wall of the cavity with small waves along the main recirculation. These waves are particularly strong for both dominant modal structures $s_{x, y}\left(\boldsymbol{r}, f_{a}\right)$ and $s_{x, y}\left(\boldsymbol{r}, f_{+}\right)$(Fig. 8a, b respectively). They correspond to strings of vorticity periodically generated by the shear layer flapping motion and injected into the cavity near the trailing edge (see Figs. 2, 3).

- When focusing on the shear layer, those modes $\left(n f_{a}, n f_{+}\right)$are structured, when considering $w_{x}$, in layers around (at least) one local minimum around the topplane of the cavity (Figs. 8, 9, 10, 11, 12c), while $w_{y}$ has a gaussian-like shape centred on the same plane around $y=0$ (Figs. 8, 9, 10, 11, 12d). This structuration in layers gets even more complex with the harmonic rank, since higher rank modes correspond to larger degrees of distorsion, as well as to smaller-scale fluctuations. As expected for Kelvin-Helmholtz waves, the wavelength of the oscillations, in the shear layer, decreases when frequency increases. For instance, $s_{y}\left(\boldsymbol{r}, 2 f_{a}\right)$ wavelength is about twice smaller than $s_{y}\left(\boldsymbol{r}, f_{a}\right)$ wavelength (see Figs. $8 \mathrm{~b}, 9 \mathrm{~b}$ ). To see examples 
Fig. 12 Spatial structure associated with first harmonic of the second most dominant mode $2 f_{+}=52.6 \mathrm{~Hz}$. Real part of a $s_{x}\left(\boldsymbol{r}, 2 f_{+}\right)$and $\mathbf{b} s_{y}\left(\boldsymbol{r}, 2 f_{+}\right)$. Power spectral density distributions $\mathbf{c} w_{x}\left(\boldsymbol{r}, 2 f_{+}\right)$and d $w_{y}\left(\boldsymbol{r}, 2 f_{+}\right)$
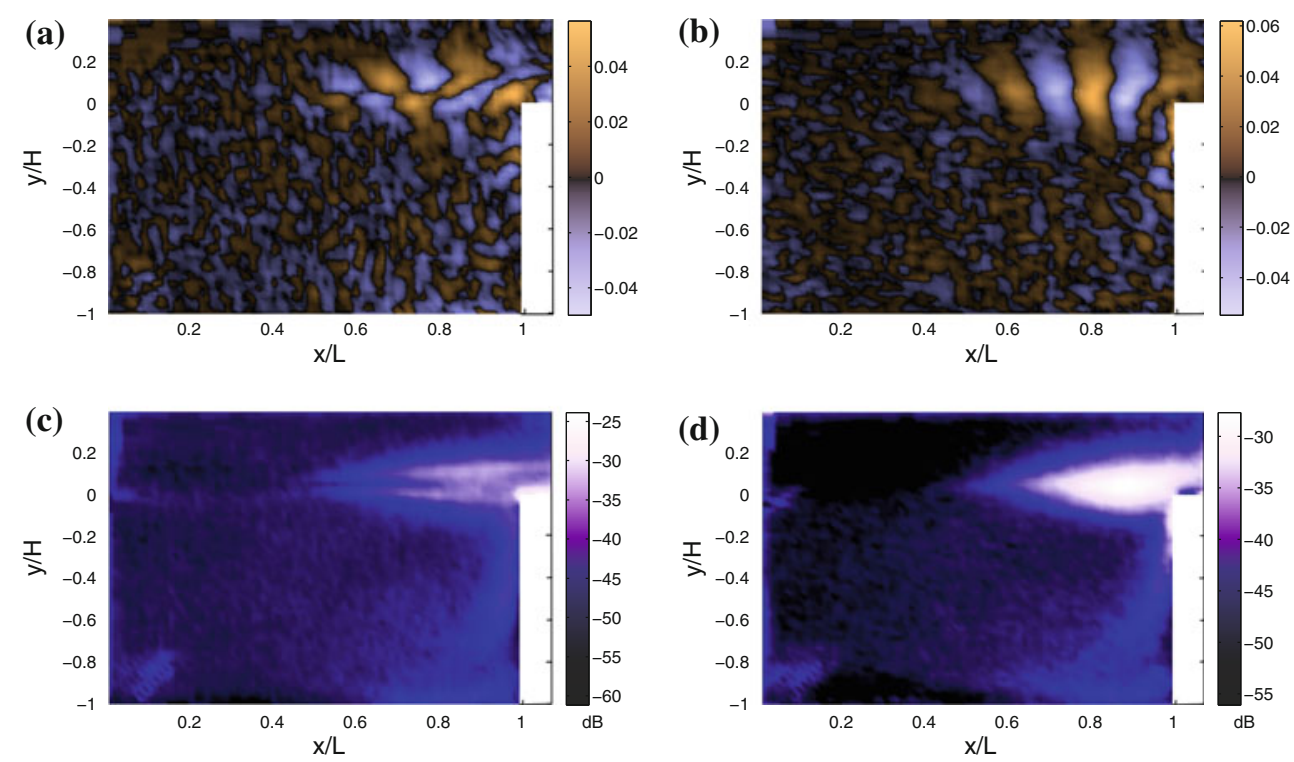

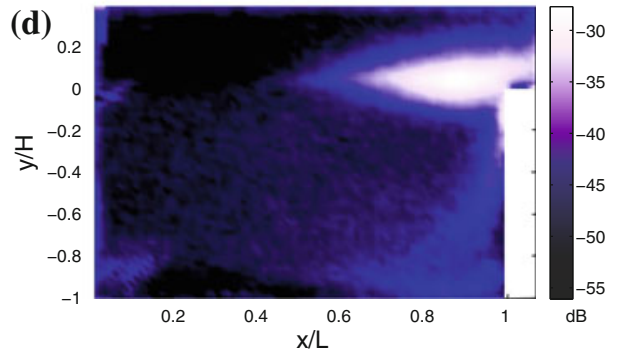

of these features in details, one can look at Fig. 13, where are shown high-resolution vector fields and vorticity colormaps in the impinging corner vicinity for both imaginary (13a) and real (13b) parts of the Fourier transform, at frequency $f_{+}$. An alley of counter-rotating eddies is easily detected and the phase-quadrature-like shift between real and imaginary parts, in the shear layer, is typical of advective structures.

- The secondary peak at $f_{2+}$, shown in Fig. $14 a-d$, looks like its corresponding $f_{a}$ harmonic modal structure, at $2 f_{a}$. Yet, its energy is much lower.

- The modal structure corresponding to the low frequency $f_{b}=6.6 \mathrm{~Hz}$ is not a shear layer mode (Fig. 15). It exhibits high amplitudes, not only at the trailing edge, but also inside the cavity. In particular, only $w_{x}\left(\boldsymbol{r}, f_{b}\right)$ (Fig. 15c) presents energy in the shear layer; amplitude in $w_{y}\left(\boldsymbol{r}, f_{b}\right)$, in this region, is small and tends to zero downstream of the cavity (Fig. 15d). On the other hand, $w_{y}\left(\boldsymbol{r}, f_{b}\right)$ exhibits a large structure entering the cavity, likely in relation with periodic vorticity injections. Moreover, the main-vortex-like distribution of $s_{x}\left(\boldsymbol{r}, f_{b}\right)$ or $s_{y}\left(\boldsymbol{r}, f_{b}\right)$ (Fig. 15a, b) or the ring-shaped structure of $w_{x}\left(\boldsymbol{r}, f_{b}\right)$, along the main recirculation (Fig. 15c), make $f_{b}$ a modal structure obviously connected to the rotating base-flow inside the cavity. This result indicates that secondary peaks $n f_{a} \pm f_{b}$ are in close connection with the recirculating flow.

In order to display the temporal behaviour of modal structures $\boldsymbol{s}\left(\boldsymbol{r}, f_{a}\right)$ and $\boldsymbol{s}\left(\boldsymbol{r}, f_{b}\right)$, a movie has been made showing both vector fields' time-evolution. Animations are obtained by multiplying each $\boldsymbol{s}(\boldsymbol{r}, f)$ to its corresponding time-complexexponential function, $\exp \left(2 i \pi f_{a} t\right)$ or $\exp \left(2 i \pi f_{b} t\right)$. The real part of the product, $\Re\{\boldsymbol{s}(\boldsymbol{r}, f) \cdot \exp (2 i \pi f t)\}$, is represented.

\subsection{Modal structures and global instability}

The modal structures associated with both $f_{a}$ and $f_{+}$harmonic families correspond to shear layer modes, as shown in Sect. 4.1. Along with their respective harmonics, they are characteristic of a global organisation of the flow. The modal structure associated with $f_{b}$ also represents a global structure, since it largely spreads all over the internal flow and the impinging region (Fig. 15). The global nature of the instability, in cavity flows, has been reported in recent works, based on numerical datasets, by Rowley et al. (2002), where DFT were also used for determining modal structures. Structures of both frequencies $f_{a}$ (Fig. 8) and $f_{+}$ (Fig. 11) strongly recall the structures of the most (linearly) unstable modes presented in Barbagallo et al. (2009), where a global-mode analysis has been carried out, in an incompressible rectangular cavity flow $(L / H=1$, $\left.R e_{L}=7500\right)$.

Theoretically, global modes are determined by investigating the linear stability of a (unstable) stationary solution of the Navier-Stokes equations. The (nonlinear) stationary solution is usually two dimensional, although threedimensional stability analysis can be performed by testing Fourier modes in the spanwise direction (most usually imposing spanwise periodic boundary conditions). The stability analysis then usually consists in a singular value decomposition of the evolution operator (Navier-Stokes), linearised around the stationary solution. Experimentally, (linear) instability actually occurs, since no stationary solution is never observed at large enough Reynolds numbers. Yet, the final state under observation may always result from the nonlinear saturation of the instability. In terms of linear stability analysis, global modes should not be easily accessible experimentally for Reynolds numbers 
Fig. 13 Full-resolution zoom on the impinging corner, for the spatial structure associated with the second most dominant mode $f_{+}=f_{a}+f_{b}=26.3 \mathrm{~Hz}$. Vorticity $\nabla \times \boldsymbol{s}\left(\boldsymbol{r}, f_{+}\right)$is displayed in color scale and normalised DFT $\boldsymbol{s}\left(\boldsymbol{r}, f_{+}\right)$as vector field. a Imaginary part and $\mathbf{b}$ real part

Fig. 14 Spatial structure associated with the second right peak $f_{2+}=2 f_{a}+f_{b}=46.0 \mathrm{~Hz}$ Real part of $\mathbf{a} s_{x}\left(\boldsymbol{r}, f_{2+}\right)$ and b $s_{y}\left(\boldsymbol{r}, f_{2+}\right)$. Power spectral density distributions $\mathbf{c} w_{x}\left(\boldsymbol{r}, f_{2+}\right)$ and $\mathbf{d} w_{y}\left(\boldsymbol{r}, f_{2+}\right)$

Fig. 15 Spatial structure associated with the lowfrequency mode $f_{b}=6.6 \mathrm{~Hz}$. Real part of $\mathbf{a} s_{x}\left(\boldsymbol{r}, f_{b}\right)$ and b $s_{y}\left(\boldsymbol{r}, f_{b}\right)$. Power spectral density distributions c $w_{x}\left(\boldsymbol{r}, f_{b}\right)$ and $\mathbf{d} w_{y}\left(\boldsymbol{r}, f_{b}\right)$
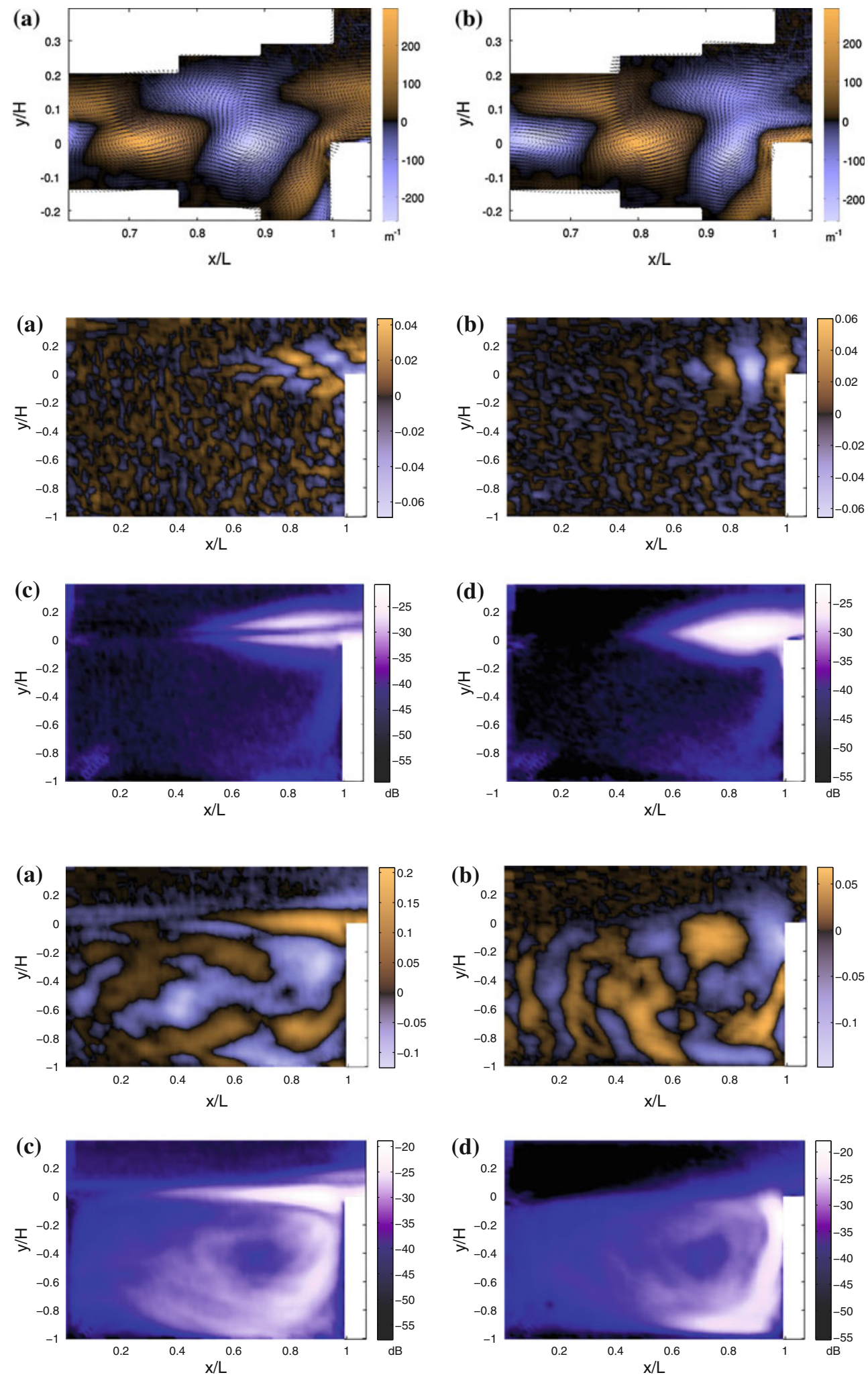

far from threshold. However, when nonlinearities are not too strong or do not distort too much the linearly growing modes, the final state might look very similar to the linearly growing global modes against which the stationary solution has become unstable. Very recently, Rowley et al. (2009) have shown that global modes are, in fact, a particular case of the so-called Koopman modes or Dynamic modes in Schmid and Sesterhenn (2008). Koopman modes are 
determined from spectral analysis of the Koopman operator, an infinite-dimensional linear operator associated with the full nonlinear system. As for global modes, Koopman modes are associated with a complex growth rate (temporal frequency and real growth rate). In the case when the dynamics is restricted to an attractor-as it is the case in our experiment-, the Koopman modes may be calculated by harmonic averages, which for finite-time datasets reduce to discrete Fourier transforms (DFT). Our modal structures, obtained by DFT on the 2D-2C time-resolved velocity field, may therefore be close to the Koopman modes of the incompressible open-cavity flow.

In the frame of a global stability analysis, harmonics may also be addressed. Indeed, higher frequency components are usually generated by nonlinear mechanisms (Knisely and Rockwell 1982). The power in $f_{a}$ is an order of magnitude larger than its first harmonic $2 f_{a}$ and two orders larger than $3 f_{a}$. As the fundamental (most unstable) mode is growing, its amplitude may become large enough to enhance its first harmonic, due to nonlinear self(squared) interaction and energy may be transferred from $f_{a}$ to $2 f_{a}$. The same applies to $3 f_{a}$, when $2 f_{a}$ has become strong enough to interact with $f_{a}$ and transfer energy to the second harmonic, and so on. If so, a global linear stability analysis may not give positive linear growth rate for harmonics $n f_{a}$, where $n \geq 2$. This scenario is further supported by the fact that the first harmonic starts to grow closer to the trailing edge than its fundamental $f_{a}$, when the amplitude of $f_{a}$ has become quite large.

\section{Conclusion}

In this study, the modal spatial structures of an incompressible cavity flow have been investigated through Fourier-transformation of velocity time-series, spatially distributed all over the 2D-2C velocity field. Such cavity spectral maps are experimentally accessible using TR-PIV techniques. Modal structures provide many new insights into organisation of incompressible cavity flows. They reveal two different shear layer modes, associated with $f_{a}$ and $f_{+}$, each composed of a harmonic family. Furthermore, modal spatial structures are assumed to be comparable to nonlinearly saturated global modes. Following Rowley et al. (2009), our modal structures should be good estimators of the Koopman modes of our (fully nonlinear) cavity flow. The shear layer modes catch both the shear layer flapping motion and vortices injection along the rotating base-flow. A mode at lower frequency, $f_{b}$, is clearly related to the internal flow, since it exhibits a modal structure on the main-vortex. Nonlinear interactions between this mode and the shear layer modes would require much interest, in particular with respect to secondary peaks related to the shear layer modes. A question indeed remains addressed about the nonlinear enhancement of some peaks in the power spectrum, such as $f_{+}$, the second most dominant frequency in the present study.

Acknowledgments This work has been supported by the ANR contracts ANR-06-BLAN-0363 "HiSpeed PIV" and ANR-07-BLAN0177 "DIB", Lionel Mathelin, Yann Fraigneau and Patrick Le Quéré are aknowledged for fruitful discussions.

Open Access This article is distributed under the terms of the Creative Commons Attribution Noncommercial License which permits any noncommercial use, distribution, and reproduction in any medium, provided the original author(s) and source are credited.

\section{References}

Alizard F, Robinet J-C (2008) Spatially convective global modes in a boundary layer. Phys Fluids 19(11):114105

Barbagallo A, Sipp D, Schmid PJ (2009) Closed-loop control of an open cavity flow using reduced-order models. J Fluid Mech 641:1-50

Brès GA, Colonius T (2008) Three-dimensional instabilities in compressible flow over open cavities. J Fluid Mech 599:309-339

Chang K, Constantinescu G, Park S-O (2006) Analysis of the flow and mass transfer processes for the incompressible flow past an open cavity with a laminar and a fully turbulent incoming boundary layer. J Fluid Mech 561:113-145

Daube O, Le Quéré P (2002) Numerical investigation of the first bifurcation for the flow in a rotor-stator cavity of radial aspect ratio 10. Comp Fluids 31:481-494

Delprat N (2006) Rossiter formula: a simple spectral model for a complex amplitude modulation process? Phys Fluids 18(7): 071703

Delprat N (2010) Low-frequency components and modulation processes in compressible cavity flows. J Sound Vib 329(22): 4797-4809

Dergham G, Brion V, Robinet J-C, Sipp D (2008) The use of global modes to compute optimal transient growth. In: 7th EUROMECH fluid mechanics conference, Manchester, England

Ehrenstein U, Gallaire F (2008) Two-dimensional global lowfrequency oscillations in a separating boundary-layer flow. J Fluid Mech 614:315-327

Faure TM, Adrianos P, Lusseyran F, Pastur LR (2007) Visualizations of the flow inside an open cavity at medium range reynolds numbers. Exp Fluids 42(2):169-184

Faure TM, Pastur LR, Lusseyran F, Fraigneau Y, Bisch D (2009) Three-dimensional centrifugal instabilities development inside a parallelepipedic open cavity of various shape. Exp Fluids 47(3): 395-410

Gadoin E, Le Quéré P, Daube O (2001) A general methodology for investigating flow instabilities in complex geometries: application to natural convection in enclosures. Int J Num Methods Fluids 37:175-208. doi:10.1002fld.173

Gallaire F, Ruith M, Meiburg E, Chomaz J-M, Huerre P (2006) Spiral vortex breakdown as a global mode. J Fluid Mech 549:71-80

Huerre P, Monkevitz PA (1985) Absolute and convective instabilities in free shear layers. J Fluid Mech 159:151-168

Huerre P, Rossi M (1998) Hydrodynamic instabilities in open flows, chapter 2. In: Godrèche C, Manneville P (eds) Hydrodynamics 
and nonlinear instabilities. Cambridge University Press, London, pp 81-294

Jackson CP (1987) A finite-element study of the onset of vortex shedding in flow past variously-shaped bodies. J Fluid Mech 182:23-45

Kegerise MA, Spina EF, Garg S, Cattafesta LN (2004) Modeswitching and nonlinear effects in compressible flow over a cavity. Phys Fluids 16:678-687

Knisely C, Rockwell D (1982) Self-sustained low-frequency components in an impinging shear layer. J Fluid Mech 116:157-186

Kuo C-H, Huang S-H (2001) Influence of flow path modification on oscillation of cavity shear layer. Exp Fluids 31:162-178

Lin JC, Rockwell D (2001) Organized oscillations of initially turbulent flow past a cavity. AIAA J 39:1139-1151

Lucas M, Rockwell D (1984) Self-excited jet—upstream modulation and multiple frequencies. J Fluid Mech 147:333-352

Mamum CK, Tuckerman LS (2009) Asymmetry and hopf bifurcation in spherical couette flow. Phys Fluids 7:80-91

Marquet O, Lombardi M, Chomaz J-M, Sipp D, Jacquin L (2009) Direct and adjoint global modes of a recirculation bubble: lift-up and convective nonnormalities. J Fluid Mech 622:1-21

Michalke A (1964) On the inviscid instability of the hyperbolictangent velocity profile. J Fluid Mech 19(4):543-556

Miksad RW (1973) Experiments on nonlinear interactions in the transition of a free shear layer. J Fluid Mech 59(1):1-21

Miksad RW, Jones FL, Powers EJ (1983) Measurements of non-linear interactions during natural transition of a symmetric wake. Phys Fluids 26(6): 1402-1409

Pastur LR, Lusseyran F, Faure TM, Pethieu R, Debesse P (2008) Quantifying the non-linear mode competition in the flow over an open cavity at medium reynolds number. Exp Fluids 44(4): 597-608
Quénot GM (1992) The orthogonal algorithm for optical flow detection using dynamic programming. In: ICASSP 3. IEEE. 249-61485, p 252

Quénot GM, Pakleza J, Kowalewski TA (1998) Particle image velocimetry with optical flow. Exp Fluids 25:177-189

Quénot GM, Rambert A, Lusseyran F, Gougat P (2001) Simple and accurate PIV camera calibration using a single target image and camera focal length. In: 4th international symposium on of particle image velocimetry. Springer, Göttingen, Germany, pp 17-19

Rockwell D (1983) Oscillations of impinging shear layers. AIAA J 21(5):645-664

Rockwell D, Knisely C (1979) The organized nature of flow impingement upon a corner. J Fluid Mech 93(3):413-432

Rockwell D, Lin JC, Oshkai P, Reiss M, Pollack M (2003) Shallow cavity flow tone experiments: onset of locked-on states. J Fluids Struct 17(3):381-414

Rockwell D, Naudascher E (1979) Self-sustained oscillations of impinging free shear layers. Annu Rev Fluid Mech 11:67-94

Rowley CW, Colonius T, Basu AJ (2002) On self-sustaining oscillations in two-dimensional compressible flow over rectangular cavitie. J Fluid Mech 455:315-346

Rowley CW, Mezic̀, Bagheri S, Schlatter P, Henningson DS (2009) Spectral analysis of nonlinear flows. J Fluid Mech 641:115-127

Schmid PJ, Sesterhenn J (2008) Dynamic mode decomposition of numerical and experimental data. In: 61st annual meeting of the APS division of fluid dynamics. APS, San Antonio, Texas, USA

Stanislas M, Okamoto M, Kähler CJ, Westerweel J (2005) 2nd international piv challenge. Exp Fluids 39:170-191

Zebib A (1987) Stability of a viscous flow past a circular cylinder. J Eng Math 21(2):155-165

Ziada S, Rockwell D (1982) Oscillations of an unstable mixing layer impinging upon an edge. J Fluid Mech 124:307-334 